\title{
Simulation of a marine dynamic positioning system equipped with cycloidal propellers
}

\author{
M. Altosole, S. Donnarumma, V. Spagnolo, S. Vignolo \\ Polytechnic School of Genoa University, Genova, Italy
}

\begin{abstract}
Marine cycloidal propellers represent a good alternative to traditional propellers especially in Dynamic Positioning (DP) applications, since they can generate almost the same thrust in all directions. The present study aims to combine a simulation platform, previously developed by some of the authors for DP systems, with the performance modelling of an epicycloidal propeller. The latter bases the propeller thrust and torque evaluation on the kinematics of the blades, taking into account suitable correction factors in order to consider the interference phenomena among blades. As a case study, the control and allocation logics of a DP system are analyzed for a surface vessel, equipped with a single bow thruster and two epicycloidal propellers at stern. The examined ship is the same for which a DP system, characterized by a conventional twin-screw propulsion, was already studied and installed on board. A performance comparison between the two distinct propulsion configurations is then carried out by dynamic simulation.
\end{abstract}

\section{INTRODUCTION}

In order to automatically maintain position and heading of a vessel, Dynamic Positioning (DP) systems employ in general waterjets or azimuth thrusters; in case, rudders and propellers (together with bow and stern thrusters) can be used too (Alessandri et al. 2014). Marine cycloidal propellers (Jürgens et al. 2002, Taniguchi 1962, Esmailian 2014), usually driven by diesel-electric propulsion to better handle the large changes in power demand (typical during DP operations), can represent a good alternative to traditional propellers since they can generate almost the same thrust in all directions. They are classified into true cycloidal, epicycloidal (e.g. Voith Schneider Propeller) and trochoidal propellers on the basis of their eccentricity value $e$, namely the ratio between the distance of the steering center from the propeller axis and the radius of the circular orbit described by the blade axes (the rotor radius): a true cycloidal propeller is characterized by $e=1$, while the conditions $e<1$ and $e>1$ distinguish epicycloidal and throcoidal propellers, respectively (Bose 2008). In the present study, the performance of an epicycloidal propeller is modelled within a DP propulsion simulator, already developed by some of the authors for a surface vessel equipped with two conventional twin-screw propellers and a bow thruster. This kind of configuration is not very suitable for station-keeping and DP applications (Sørensen 1996, Sørensen 2011, Fossen 1996, Fossen 2002), never- theless, a conventional propulsion configuration could be requested for specific operations characterized by limited DP capabilities. For instance, the mentioned simulator was developed for a patrol vessel designed with a twin propeller-rudder configuration and a single bow-thruster, which were requested to provide a certain dynamic positioning performance at zero-speed with moderate weather conditions. The main purpose of the DP simulation model was to validate the Force and Thrust Allocation Logic (FAL, TAL, Johansen 2013), specifically designed for such propulsion configuration (Donnarumma et al. 2015).

In this new work, the same vessel, but supposed equipped with a single bow thruster and two epicycloidal propellers at stern, is simulated in order to analyze the main differences during DP operations, in terms of general performance and control system behavior. This kind of simulation involves a reliable representation of the epicycloidal propellers, whose manufacturers unfortunately do not publicly share their performance maps for confidential reasons. Therefore, simplified simulation approaches, as possible for traditional propellers (Altosole et al. 2012, Martelli 2015) or waterjets (Altosole et al. 2005), are quite difficult to be developed. The present numerical modelling is based on a mixture of theoretical and empirical considerations: in particular, the propeller thrust and torque evaluation is based on the kinematics of the blades, taking into account suitable correction factors in order to con- 
sider the interference phenomena among blades. The result is a simulation approach able to predict the performance of an epicycloidal propeller, avoiding demanding computations (e.g. CFD methods) that would not allow an effective simulation of the whole DP system.

\section{DP SIMULATION MODEL}

Figure 1 provides a sketch of the devised DP-logic circuit, specific for the target ship. The system block simulates, through the equations of motion, the presence of a Positioning Reference System (PRS), composed by a DGPS and a Fiber Optic Gyro (FOG), which picks up the instantaneous position and velocity of the vessel. Such measurements are compared with the corresponding desired quantities in order to compute the position and velocity errors.

The circuit extrapolates the low-frequency (LF) components of the errors and send them to the regulator, where the required forces and moments are firstly evaluated and subsequently allocated to the actuators. Environment action and delivered forces are then used within the equations of motion to obtain the new position and velocity.

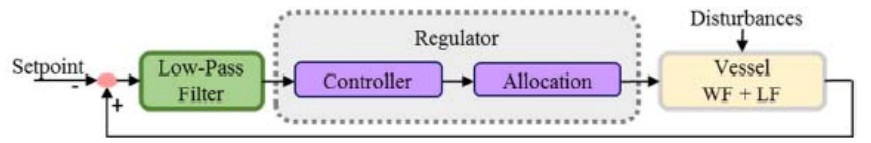

Figure 1. DP simulation model.

The mathematical models adopted for ship motions and for wind, wave, and current forces, as well as for the controller, are briefly illustrated below.

\subsection{Ship motions}

Setting $\eta:=[x, y, \psi]^{T} \in \mathbb{R}^{3}$, the array of the position (longitudinal and lateral position and orientation) of the vessel w.r.t. the Earth-fixed frame, and $v:=[u, v, r]^{T} \in \mathbb{R}^{3}$, the array of the components of velocity (linear and angular) expressed in the bodyfixed basis, the ship kinematics is described by the relations:

$\dot{\eta}=R(\psi) v, \quad R(\psi)=\left(\begin{array}{ccc}\cos \psi & -\sin \psi & 0 \\ \sin \psi & \cos \psi & 0 \\ 0 & 0 & 1\end{array}\right)$

The ship motion equations are given by:

$M \dot{v}+C(v) v+D_{0} v+D(v) v=\tau_{D}+\tau_{E}$

where $M, C$ and $D$ are mass-inertia and added mass, Coriolis and damping matrices respectively, the array $\tau:=[X, Y, N]^{T} \in \mathbb{R}^{3}$ represents the components of the resultant force and moment ( $\tau_{D}$ for delivered and $\tau_{E}$ for environmental forces and moments), expressed in the body-fixed basis.

\subsection{Environmental forces and moments}

Environmental disturbances are evaluated as the sum of forces and moments due to wind, current and wave respectively. Forces and moments are expressed making use of the well-known resistance form, depending on non-dimensional coefficients $\mathrm{C}_{\mathrm{X}}, \mathrm{C}_{\mathrm{Y}}$, and $\mathrm{C}_{\mathrm{N}}$, related respectively to the longitudinal force, the lateral force and the moment. In order to consider the occurring worst condition, all environmental disturbances are supposed to be aligned in the same incoming direction. The current and the wind speeds are assumed constant and wave drift forces are modelled as proportional to the square of the significant height $H_{s}$. Collecting all the (bodyfixed basis) components of the force and moment in a unique 3-dimensional array $\tau$, we have:

$$
\tau_{\mathrm{E}}=\tau_{\text {current }}+\tau_{\text {waves }}+\tau_{\text {wind }}
$$

\subsection{Controller}

The controller consists of a PD (proportional and derivative controller), a wind forces reconstruction, a sea force estimation and a block for allocation logic (Figure 2).

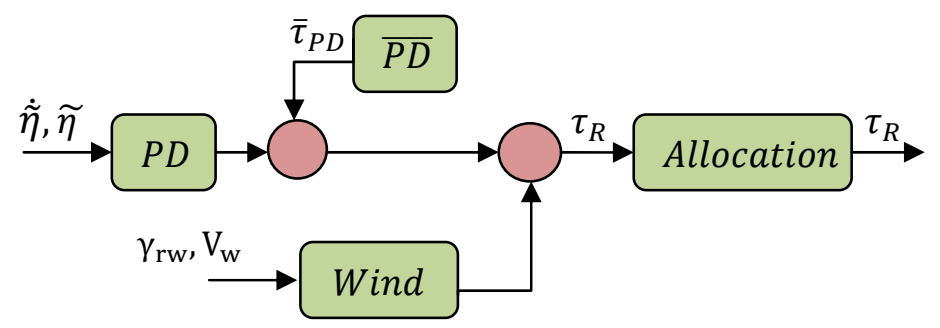

Figure 2. Controller Layout.

The controller law is given by:

$\tau_{\mathrm{R}}=K_{P} \tilde{\eta}+K_{D} \dot{\tilde{\eta}}+\bar{\tau}_{P D}-\tau_{W}$

where the output $\tau_{\mathrm{R}}$ represents the required force and moment; in Eq. (4), $K_{P}$ and $K_{D}$ are constant matrices, $\bar{\tau}_{P D}$ and $\tau_{W}$ are contributions which compensate the environmental disturbances (Donnarumma et al. in press) and the quantities $\tilde{\eta}:=\eta-\eta_{d}$ and $\dot{\tilde{\eta}}:=\dot{\eta}-\dot{\eta}_{d}$ are controller input errors, $\eta_{d}$ and $\dot{\eta}_{d}$ denoting the desired position and velocity respectively.

\subsection{Allocation}

The adopted thrust allocation logic (TAL) is based on a constrained minimum problem. The idea is to minimize a cost function of the seven variables

$\underline{x}=\left[T_{p t}, T_{s b}, T_{b o w}, X_{p t}, Y_{p t}, X_{s b}, Y_{s b}\right] \in \mathbb{R}^{7}$

subjected to some suitable constraints. In particular, denoting by $T_{p t}$ and $T_{s b}$ the portside and starboard thrusts respectively, $T_{\text {bow }}$ the thrust of the bow thruster, $\left(X_{p t}, Y_{p t}\right)$ and $\left(X_{s b}, Y_{s b}\right)$ the components of the portside and starboard thrust forces in the body- 
fixed basis, the constrained minimum problem is formulated as

$\min _{\underline{x}} f(\underline{x})$ with $h_{\mathrm{i}}(\underline{x})=0 ; g_{\mathrm{j}}(\underline{x})>0$

where

$f(\underline{x})=\left(\frac{T_{\mathrm{pt}}}{T_{\max }^{\text {tot }}}\right)^{2}+\left(\frac{T_{\mathrm{sb}}}{T_{\max }^{\text {tot }}}\right)^{2}+\left(\frac{T_{\mathrm{bow}}}{T_{\max }^{\text {tot }}}\right)^{2}$

is the cost function to be minimized, and

$\left\{\begin{array}{c}h_{1}(\underline{x})=X_{e n v}-X_{p t}-X_{s b}=0 \\ h_{2}(\underline{x})=Y_{e n v}-Y_{p t}-Y_{s b}-T_{b o w}=0 \\ h_{3}(\underline{x})=N_{e n v}-x_{b o w} T_{b o w}-x_{p t} Y_{p t}+y_{p t} X_{p t}+ \\ \quad-x_{s b} Y_{s b}+y_{s b} X_{s b}=0 \\ h_{4}(\underline{x})=T_{p t}^{2}-X_{p t}^{2}-Y_{p t}^{2}=0 \\ h_{5}(\underline{x})=T_{s b}^{2}-X_{s b}^{2}-Y_{s b}^{2}=0\end{array}\right.$

are the constraints to be satisfied; in Eq. (8), $\left\{X_{e n v}, Y_{e n v}, N_{e n v}\right\}$ are the components of the force and the moment due to environmental disturbances, $\left(x_{p t}, y_{p t}\right)$ and $\left(x_{s b}, y_{s b}\right)$ are the coordinates of the propellers thrust centres and $x_{\text {bow }}$ is the longitudinal coordinate of the bow thruster. Moreover, we have

$g_{1}(\underline{x})=T_{p t}>0 \quad, g_{2}(\underline{x})=T_{s b}>0$

Eq. (6) and (7) require that the sum of the squared desired thrusts is minimum. Eq. (8) details the constraints: the first three represent the equilibrium between the environmental disturbances and the delivered force and moment; the last two correlate the modulus of the portside and starboard thrust forces with their longitudinal and lateral components. Finally, Eq. (9) ensures that the modulus of the two aft thrusts is positive.

\section{EPICYCLOIDAL PROPELLER MODEL}

\subsection{Kinematics}

In this subsection, we sketch the kinematical model adopted to describe the motion of the blades of a given epicycloidal propeller. Such 2-dimensional plane model makes use of two distinguished reference frames (see Figure 3): the first one $\left(0, \underline{b}_{1}, \underline{b}_{2}, \underline{b}_{3}\right)$ is fixed to the hull and it has its origin $O$ at the center of the rotor, the unit vector $\underline{b}_{1}$ points towards the bow, the unit vector $\underline{b}_{2}$ points towards starboard and the unit vector $\underline{b}_{3}=\underline{b}_{1} \wedge \underline{b}_{2}$ points downwards; the second one $\left(O, \underline{e}_{1}, \underline{e}_{2}, \underline{e}_{3}\right)$ rotates clockwise about the vertical axis passing through $O$ and parallel to $\underline{b}_{3}=\underline{e}_{3}$, by an angle $\beta \epsilon[0,2 \pi]$ which determines (the perpendicular of) the steering force direction. The angle $\beta$ is related to the rudder pitch of the epicycloidal propeller. The steering centre $C$ lies on the straight line passing through $O$ and parallel to $e_{2}$. During the revolution motion, the projection $P$ of the blade shaft on the plane $\left\langle O, \underline{b}_{1}, \underline{b}_{2}\right\rangle$ de- scribes a circumference having centre $O$ and radius $R$, coinciding with the rotor radius. We parameterize such a circumference by an angle $\theta$ (function of time), in such a way that the unit tangent vector $t$ is expressed as $\underline{t}(\theta)=-\sin \theta \underline{b}_{1}+\cos \theta \underline{b}_{2}$. Introducing the vector $(C-O)=s \underline{e}_{2}=-s \sin \beta \underline{b}_{1}+$ $s \cos \beta \underline{b}_{2}$ (with $s \in[0,0.8 R]$ ), the vector joining the steering centre $C$ with the point $P$ can be expressed as $\quad(P-C)=(R \cos \theta+s \sin \beta) \underline{b}_{1}+(R \sin \theta-$ $s \cos \beta) \underline{b}_{2}$. The variable $s$ is usually called driving pitch and it controls the magnitude of the thrust. The unit vector $\frac{(P-C)^{\perp}}{\left|(P-C)^{\perp}\right|}$, orthogonal to $(P-C)$ and belonging to the plane $\left\langle O, \underline{b}_{1}, \underline{b}_{2}\right\rangle$, identifies with the unit vector of the blade chord. Therefore, the pivoting motion of the blade around its shaft can be described by the angle $\alpha$ (function of time) between the unit vectors $\underline{t}$ and $\frac{(P-C)^{\perp}}{\left|(P-C)^{\perp}\right|}$. Choosing anticlockwise the positive direction of rotation around the blade shaft, the pivoting angle $\alpha$ can be defined as

$\alpha=\left\{\begin{array}{lr}\cos ^{-1}\left(\frac{(P-C)^{\perp}}{\mid(P-C)^{\perp}} \cdot \underline{t}\right) & \text { if } \cos (\theta-\beta) \geq 0 \\ -\cos ^{-1}\left(\frac{(P-C)^{\perp}}{\left|(P-C)^{\perp}\right|} \cdot \underline{t}\right) & \text { otherwise }\end{array}\right.$

where

$$
\begin{aligned}
& \frac{(P-C)^{\perp}}{\left|(P-C)^{\perp}\right|} \cdot \underline{t} \\
& =\frac{R+s \sin (\beta-\theta)}{\sqrt{(-R \sin \theta+s \cos \beta)^{2}+(R \cos \theta+s \sin \beta)^{2}}} \\
& \quad=\cos \alpha
\end{aligned}
$$

Figure 3 summarizes the above outlined kinematical scheme.

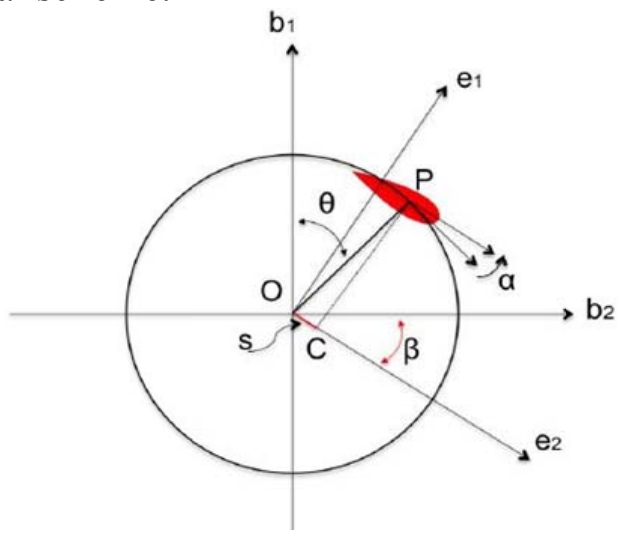

Figure 3. Kinematics of the blade.

Supposing now that the vessel is moving, let $\underline{v}_{0}=\hat{u} \underline{b}_{1}+\hat{v} \underline{b}_{2}$ be the velocity of $O$ (w.r.t. the Earth-fixed frame) expressed in the hull-fixed basis. Denoting by $\underline{v}_{P}^{\prime}=-R \dot{\theta} \sin \theta \underline{b}_{1}+R \dot{\theta} \cos \theta \underline{b}_{2}$ the velocity of the point $P$ w.r.t. the body-fixed frame, 
the velocity of $\mathrm{P}$ w.r.t. the Earth-fixed frame is given by

$$
\underline{v}_{P}=\underline{v}_{P}^{\prime}+\underline{v}_{0}+\underline{\omega}(P-O)=[\hat{u}-R(\dot{\theta}+
$$

$r \sin \theta b 1+v+R \theta+r \cos \theta b 2$

where $\underline{\omega}=r \underline{b}_{3}$ is the angular velocity of the vessel. The velocity of the incoming flow experienced at $P$ by a blade-fixed observer is then $-\underline{v}_{P}$; its unit vector $\hat{t}$ is expressed as

$$
\begin{gathered}
\hat{t}=-\frac{\underline{v}_{P}}{\left|\underline{v}_{P}\right|}= \\
-\frac{[\hat{u}-R(\dot{\theta}+r) \sin \theta] \underline{b_{1}}+[\hat{v}+R(\dot{\theta}+r) \cos \theta] \underline{b_{2}}}{\sqrt{[\hat{u}-R(\dot{\theta}+r) \sin \theta]^{2}+[\hat{v}+R(\dot{\theta}+r) \cos \theta]^{2}}}
\end{gathered}
$$

Making use of the unit vector $\underline{\hat{t}}$, it is possible to characterize the attack angle of the incident flow as

$$
\hat{\alpha}=\pi-\cos ^{-1}\left[\frac{(P-C)^{\perp}}{\left|(P-C)^{\perp}\right|} \cdot \hat{t}\right]
$$

according to Figure 4.

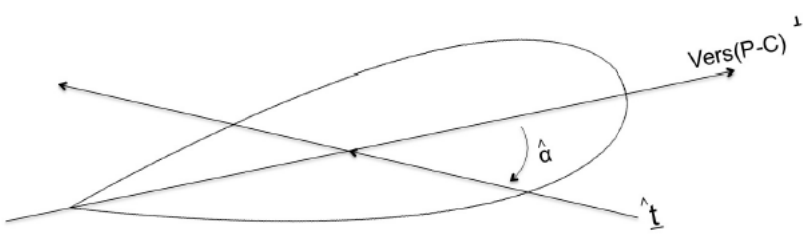

Figure 4. Angle of attack.

\subsection{Hydrodynamic forces}

In this subsection, making use of some simplifying assumptions, we present a simple model for evaluating the hydrodynamic forces generated by each blade. It is supposed that the velocity of the incident flow be the same over the entire surface of the blade and coincide with $-\underline{v}_{P}$. Under such a condition, the lift and drag produced by each blade can be expressed as

$$
\begin{aligned}
& \underline{L}=c_{L} \frac{1}{2} \rho_{w} A\left|\underline{v}_{P}\right|^{2} \underline{\hat{n}} \\
& \underline{D}=c_{D} \frac{1}{2} \rho_{w} A\left|\underline{v}_{P}\right|^{2} \underline{\hat{t}}
\end{aligned}
$$

where $c_{L}$ is the lift coefficient, $c_{D}$ is the drag coefficient, $\rho_{w}$ is sea water density, $A$ is the blade lateral area, $\left|\underline{v}_{P}\right|$ is the incoming flow speed, $\hat{t}$ is the unit vector of the lift force (unit vector of the incoming flow at $P$ ), and $\underline{\hat{n}}$ is the unit vector of the drag force (perpendicular to $\underline{\hat{t}}$ ). The unit vector $\underline{\hat{n}}$ can be determined by the following procedure, in which two main scenarios are distinguished:

- $\quad$ the attack angle $\hat{\alpha}$ belongs to the interval $] 0, \frac{\pi}{2}[$, namely the incoming flow hits the blade from the front. In such a circumstance, the unit vector $\underline{\hat{n}}$ is determined according to the requirements:

$$
\underline{\hat{n}}= \begin{cases}\underline{b}_{3} \wedge \underline{\hat{t}} & \text { when } \underline{\hat{t}} \wedge \frac{(P-C)^{\perp}}{\left|(P-C)^{\perp}\right|} \cdot \underline{b}_{3}>0 \\ -\underline{b}_{3} \wedge \underline{\hat{t}} & \text { when } \underline{\hat{t}} \wedge \frac{(P-C)^{\perp}}{\left|(P-C)^{\perp}\right|} \cdot \underline{b}_{3}<0\end{cases}
$$

- $\hat{\alpha} \epsilon] \frac{\pi}{2}, \pi[$, the incoming flow hits the blade from the back. In this case, $\underline{\hat{n}}$ is singled out by the requests:

$$
\underline{\hat{n}}= \begin{cases}-\underline{b}_{3} \wedge \underline{\hat{t}} & \text { when } \underline{\hat{t}} \wedge \frac{(P-C)^{\perp}}{\left|(P-C)^{\perp}\right|} \cdot \underline{b}_{3}>0 \\ \underline{b}_{3} \wedge \underline{\hat{t}} & \text { when } \underline{\hat{t}} \wedge \frac{(P-C)^{\perp}}{\left|(P-C)^{\perp}\right|} \cdot \underline{b}_{3}<0\end{cases}
$$

As remaining particular cases, if $\hat{\alpha}=0$ or $\hat{\alpha}=\pi$ there is no lift while if $\hat{\alpha}=\frac{\pi}{2}$ then $\underline{\hat{n}}=\underline{\hat{t}}$. The above described procedure allows to determine the lift and drag provided by each single blade. The resultant hydrodynamic force generated by the epicycloidal propeller can be computed as the sum of all contributions given by each blade.

\subsection{Torque acting on the rotor}

In order to calculate the torque acting on the rotor, the Newton-Euler moment equations for each single blade and for the rotor are considered separately. Developed in the hull-fixed reference frame and with respect to the point $O$ (center of the rotor), the Newton-Euler moments equation for each blade can be expressed as

$$
\begin{aligned}
& \underline{M}_{O}^{H}+\underline{M}_{O}^{G}+\underline{M}_{O}^{R}+\underline{M}_{O}^{I}=I_{G}(\underline{\dot{\omega}})+\underline{\omega} \wedge I_{G}(\underline{\omega})+ \\
&+m(G-O) \wedge \underline{a}_{G}
\end{aligned}
$$

where, $\underline{M}_{O}^{G}, \underline{M}_{O}^{H}, \underline{M}_{O}^{R}$, and $\underline{M}_{O}^{I}$ are respectively the hydrodynamic, weight force, reactive force, and inertial force torques w.r.t. $O$ acting on the blade; $I_{G}$ is the inertia tensor w.r.t. the center gravity $G$ of the blade; $\underline{\omega}=(\dot{\theta}-\dot{\alpha}) \underline{b}_{3}$ is the blade angular velocity w.r.t. the hull-fixed frame; $\underline{a}_{G}$ is the acceleration of $G$ w.r.t. the hull-fixed frame; and $m$ is the blade mass.

Knowing the revolution velocity of the rotor and the position of the steering center as well as the velocity of the incoming flow, the consequent motion of the blade is known from kinematics; at the same time, the knowledge of the hydrodynamic forces allows the evaluation of their moment. Evaluating the reactive torques from Eq. (19) and inserting them in the moment equation for the rotor, the (scalar value of) engine torque can be calculated as $M_{O}^{E}=\sum_{i=1}^{n}\left(\underline{M}_{O}^{R}\right)_{i} \cdot \underline{b}_{3}-\underline{M}_{O}^{I} \cdot \underline{b}_{3}+I_{O}\left(\underline{\dot{\omega}}_{r}\right) \cdot \underline{b}_{3}$

where $\underline{M}_{O}^{I}$ is the inertial forces torque acting on the rotor, $I_{O}$ is the inertia tensor of the rotor and $\underline{\omega}_{r}=\dot{\theta} \underline{b}_{3}$ is the angular velocity of the rotor, $\mathrm{n}$ is the number of blades. 


\subsection{Validation and thrust generation}

The main features and the validation of the simulator based on the mathematical model illustrated above have been presented in Altosole at al. (2017). For sake of shortness, we only recall that the interference among the blades is taken into account by means of three correction factors validated with the open water diagram of an existing propeller: shielding correction, referring to the shielding of the blades that are in the half circumference not directly exposed to the incoming flow (in the model, the correction factor, depending on driving pitch values, reduces the right velocity of the incoming water flow); interference correction, modeled by reducing the attack angle of the incoming flow with respect to the chord of the blade section (the correction depends on the advance coefficient and pitch values); reverse thrust correction, representing the reduction of the reverse thrust (comparing butterfly diagrams found in open source with those obtained by simulation, we found out that for advance coefficient more than 0.4 there was a reduction of the thrust when the steering pitch $\beta$ was between $\pi / 2$ and $3 \pi / 2$ : we introduced this coefficient to take into account this further phenomenon).

As we have illustrated in the description of the epicycloidal propeller model, there are two different pitches $(s$ and $\beta$ ) that control the magnitude and the direction of the thrust, together with the choice of a suitable rpm. In this case study, simulations have been made by keeping rpm constant, so modifying the thrusts only by means of the two geometric pitches. When the simulator runs, the required thrusts are translated in terms of corresponding control pitches and thus the delivered thrusts are generated: the steering pitch $\beta$ is strictly linked to direction of the required thrust (aligned along the unit vector $e_{1}$, see Figure 3 ), while the other pitch $\mathrm{s}$ is uniquely determined by a matching algorithm that combines a given required thrust (at fixed rpm) to a predetermined geometric pitch.

\section{SIMULATION RESULTS}

In this section, some simulation results concerning the vessel equipped with epicycloidal propellers are presented and compared with those obtained by previous simulations of the same vessel, equipped with a conventional twin screw propulsion system.

The environmental disturbances have been modeled as detailed in Alessandri et al. (2014) and briefly recalled in Subsection 2.2. In order to consider the worst environmental condition, the disturbances (sea, wind and current) are considered aligned and coming from the same direction. For this work we maintained Mediterranean SS 4 (significant wave height of $1.8 \mathrm{~m}$, wave period of $8.8 \mathrm{~s}$ ) and a constant current speed of $1 \mathrm{kn}$ as for the DP capability plots presented in (Donnarumma et al., in press), where a static analysis of the ship performance has been presented.

We show the simulation results in the presence of environmental disturbances coming from an angle of $30^{\circ}$ with respect to the desired heading, in two distinct cases: $10 \mathrm{kn}$ and $30 \mathrm{kn}$ wind speed. Consistently with what proposed in Donnarumma et al. (in press), the evaluation of the environmental disturbances mean components $\bar{\tau}_{P D}$ and $\tau_{W}$ requires some minutes of transient that are not relevant for the station keeping performances evaluation. For such a reason, first few minutes of simulation have been neglected. In Figures 5, 6 and 7 the variations of the ship position and heading are shown in the two different environmental conditions. As we can see, for a wind speed of $10 \mathrm{kn}$ both the propulsion configurations are able to keep the desired position and heading; however, different amplitudes of the oscillations around the desired set-point and then different performances of the two propulsion systems are evident. For a wind speed of $30 \mathrm{kn}$ instead, the conventional propulsion cannot perform the desired DP maneuver (see Figure 6). The same conclusions are reflected in the Figures from 8 to 13, where differences between the two propulsion plants are underlined. The thrusts required to the actuators and thus delivered by the two propulsion plants are very far from each other. Also the required and delivered force and moment are deeply unequal, since the deviation from the desired set-point is very different. These results are reflected in Figures 12 and 13, where the power required to the engines for the two propulsion configurations is shown.

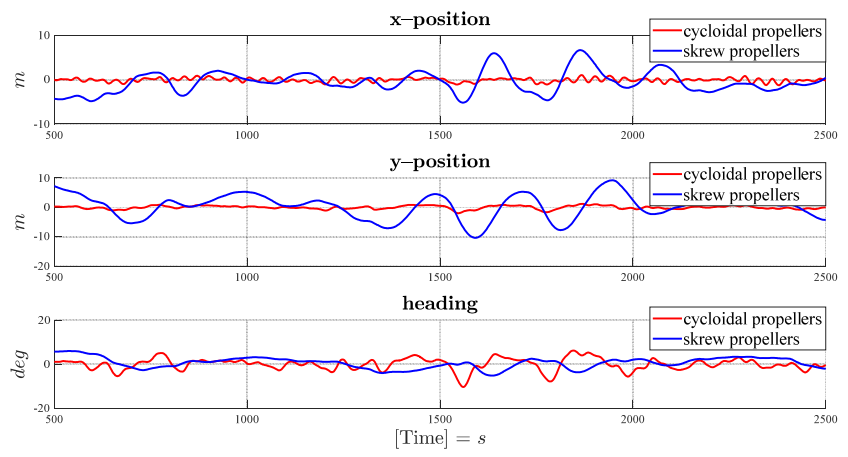

Figure 5. Motions time history for wind speed of $10 \mathrm{kn}$.
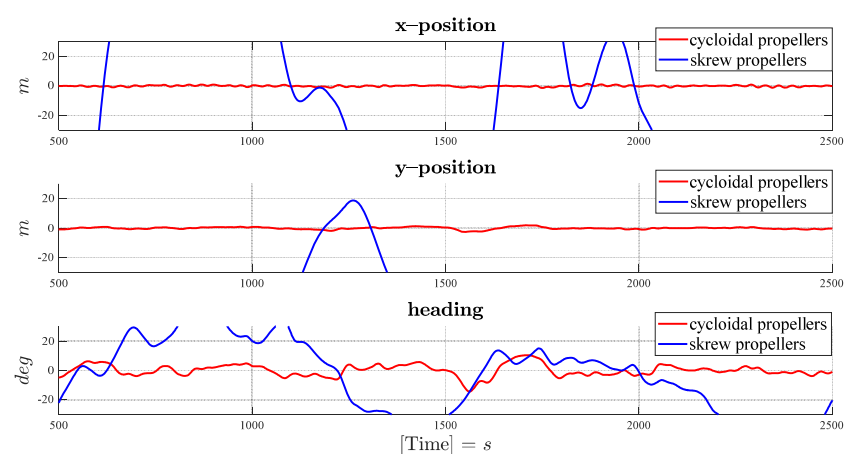

Figure 6. Motions time history for wind speed of $30 \mathrm{kn}$. 

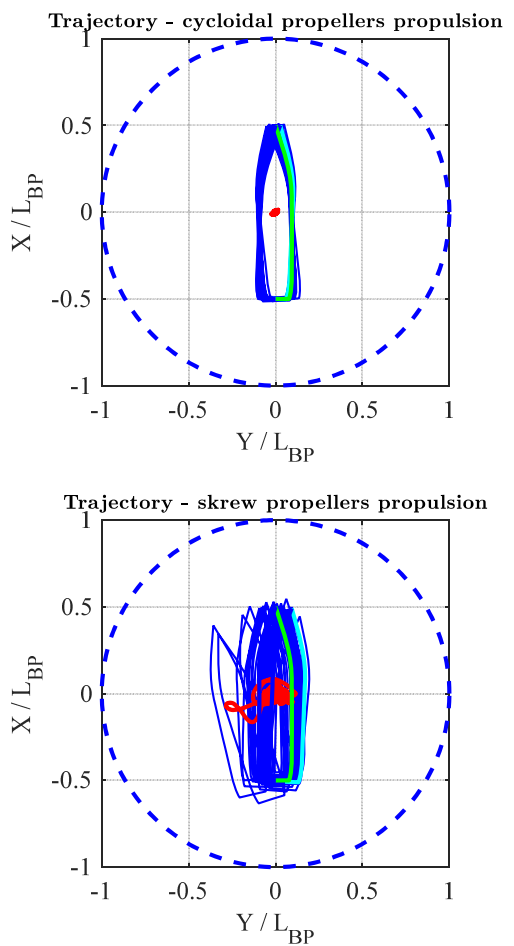
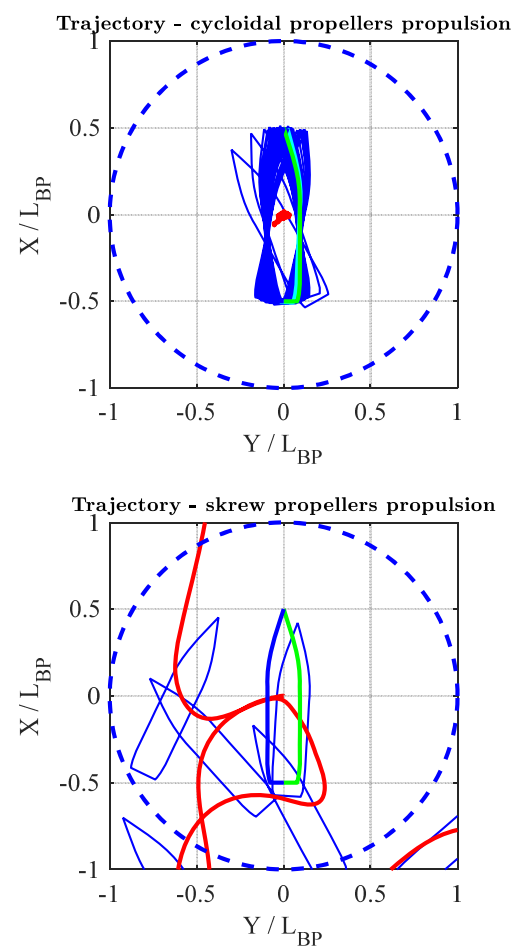

Figure 7. Ship position (trajectory of the origin of the body-fixed frame) and orientation variations for wind $10 \mathrm{kn}$ (on the left) and $30 \mathrm{kn}$ (on the right).
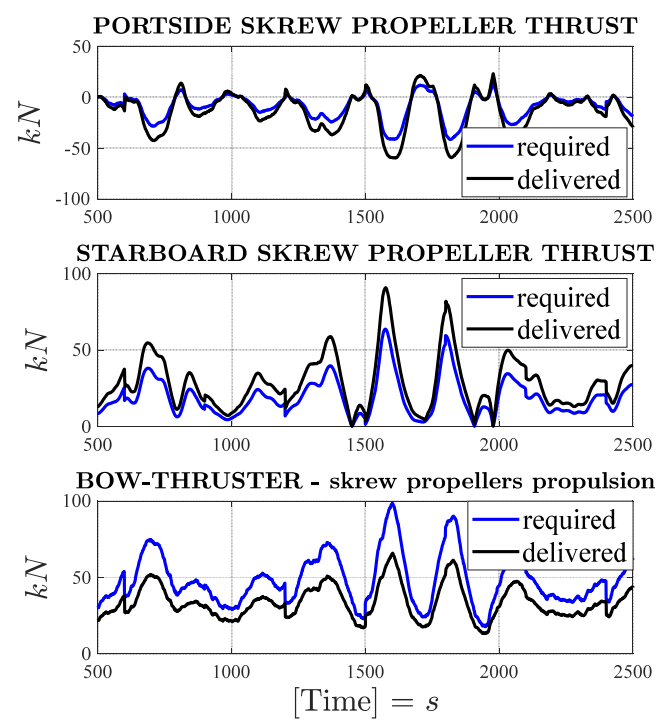

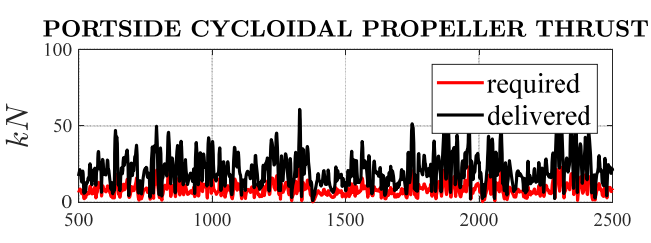

STARBOARD CYCLOIDAL PROPELLER THRUST

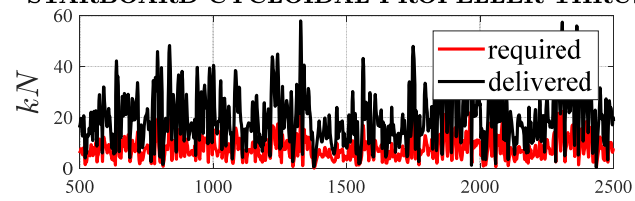

BOW-THRUSTER - cycloidal propellers propulsion

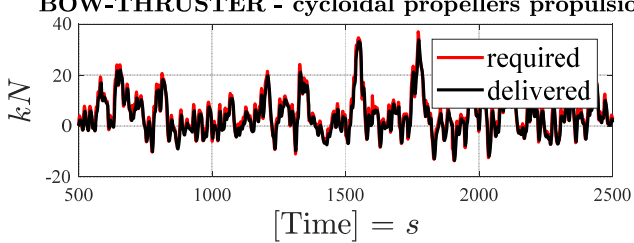

Figure 8 . Time history of required and delivered thrust for wind speed of $10 \mathrm{kn}$.

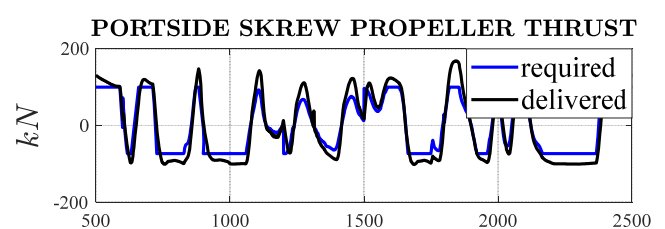

STARBOARD SKREW PROPELLER THRUST

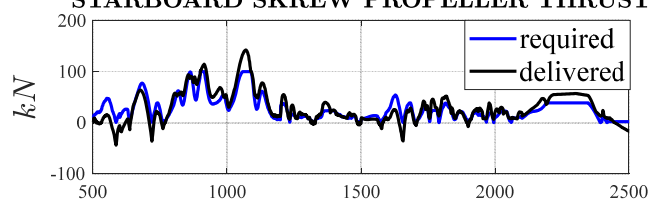

BOW-THRUSTER - skrew propellers propulsion

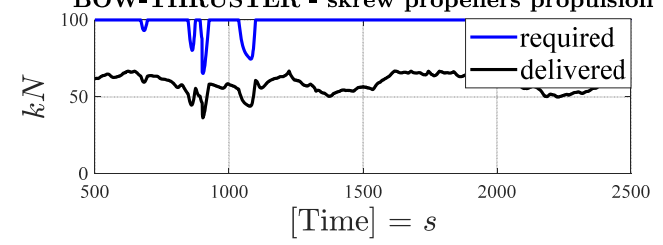

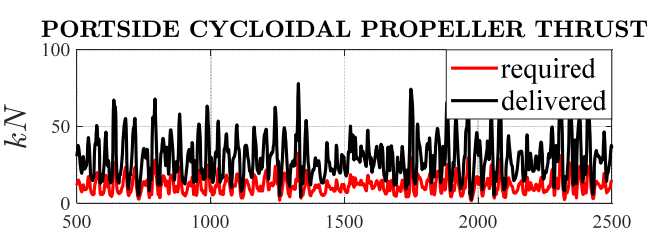

STARBOARD CYCLOIDAL PROPELLER THRUST
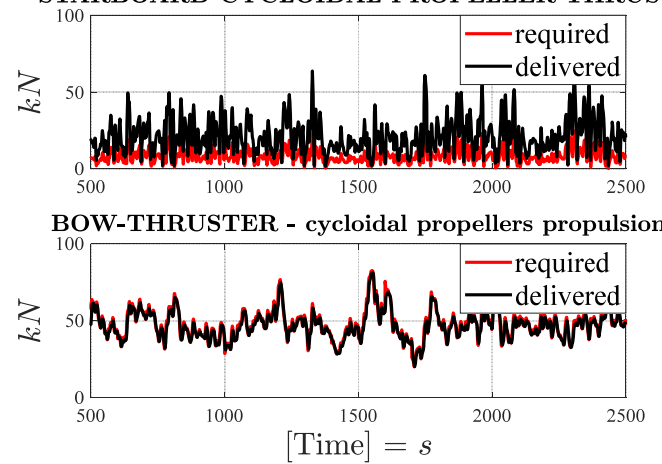

Figure 9. Time history of required and delivered thrust for wind speed of $30 \mathrm{kn}$. 

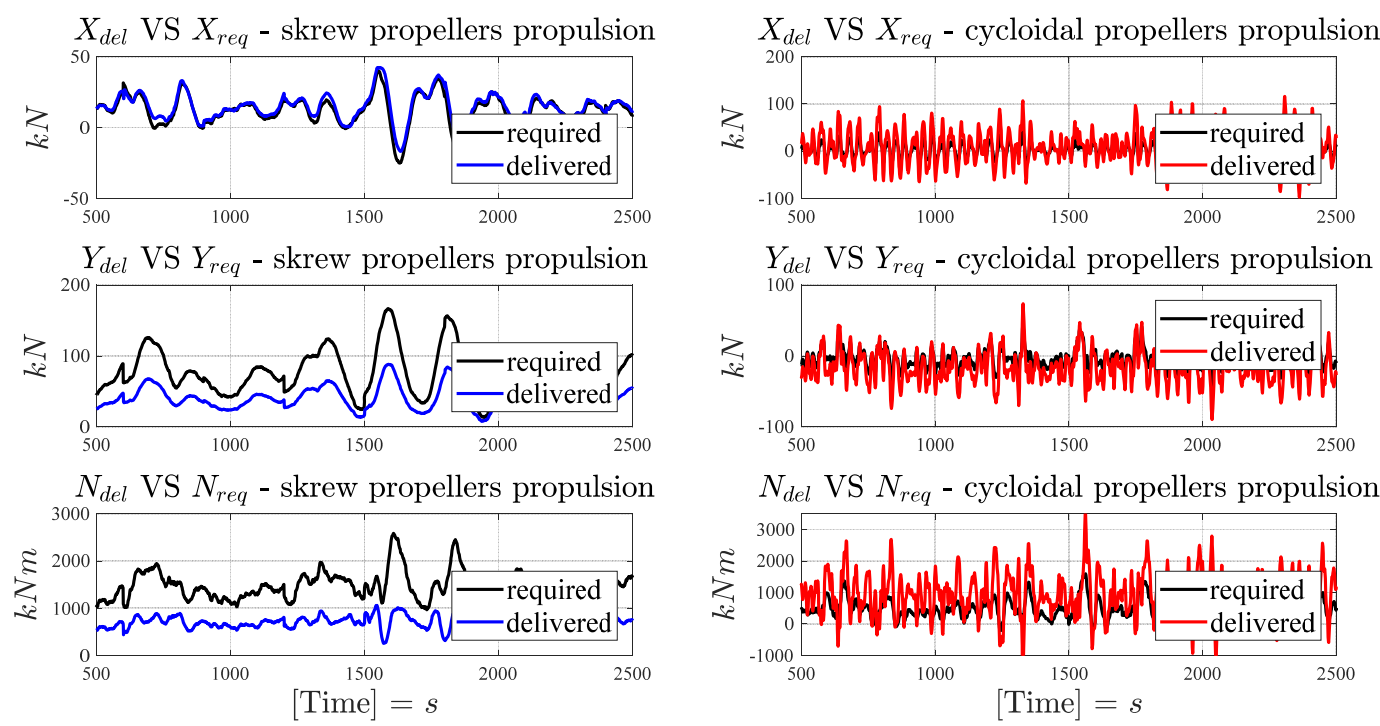

Figure 10. Time history of required and delivered force and moment for wind speed of $10 \mathrm{kn}$.
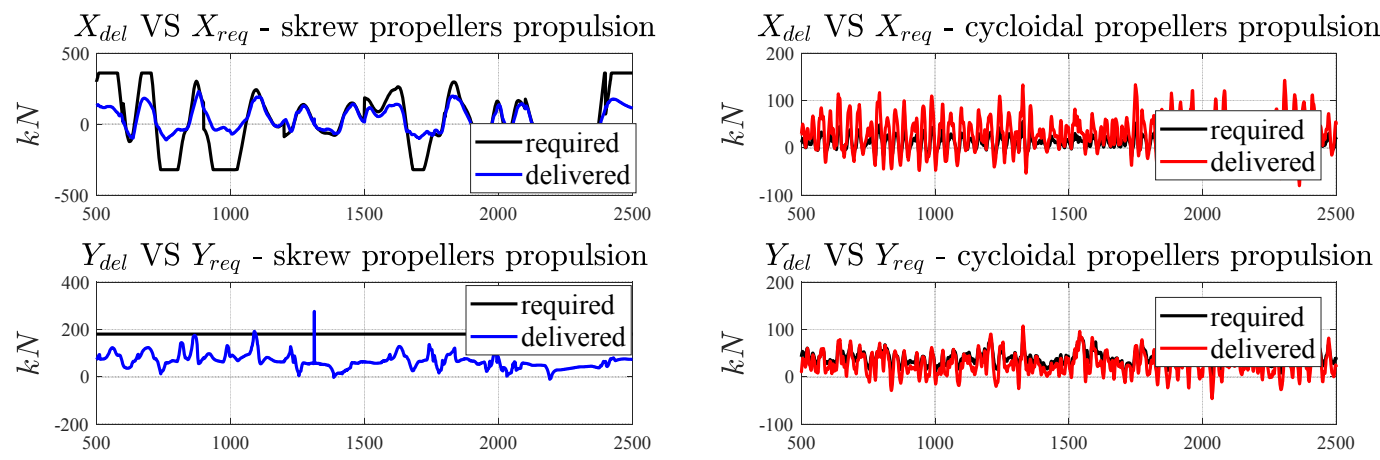

$Y_{\text {del }}$ VS $Y_{\text {req }}$ - cycloidal propellers propulsion
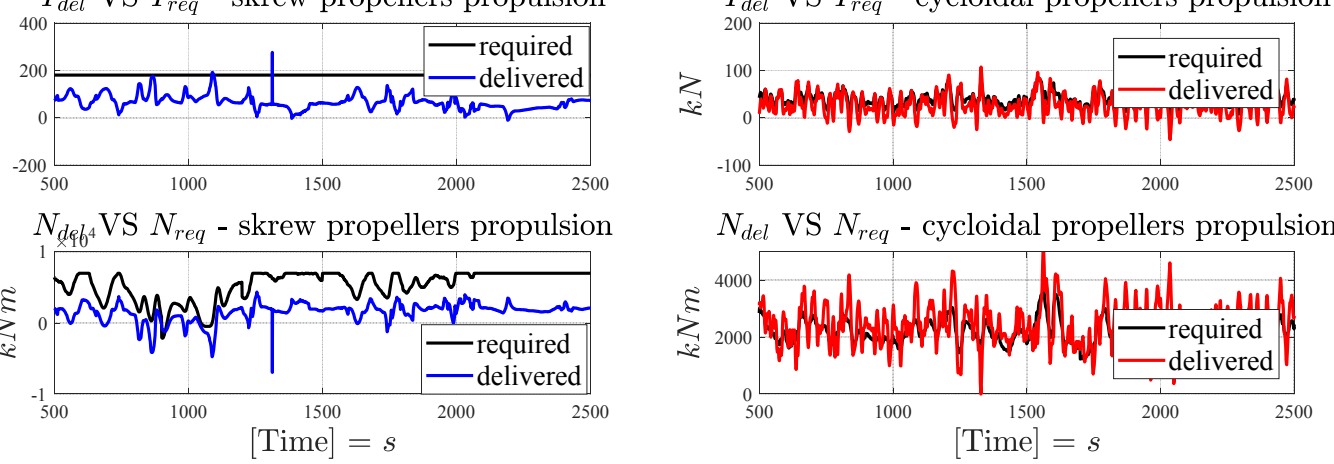

Figure 11. Time history of required and delivered force and moment for wind speed of $30 \mathrm{kn}$.
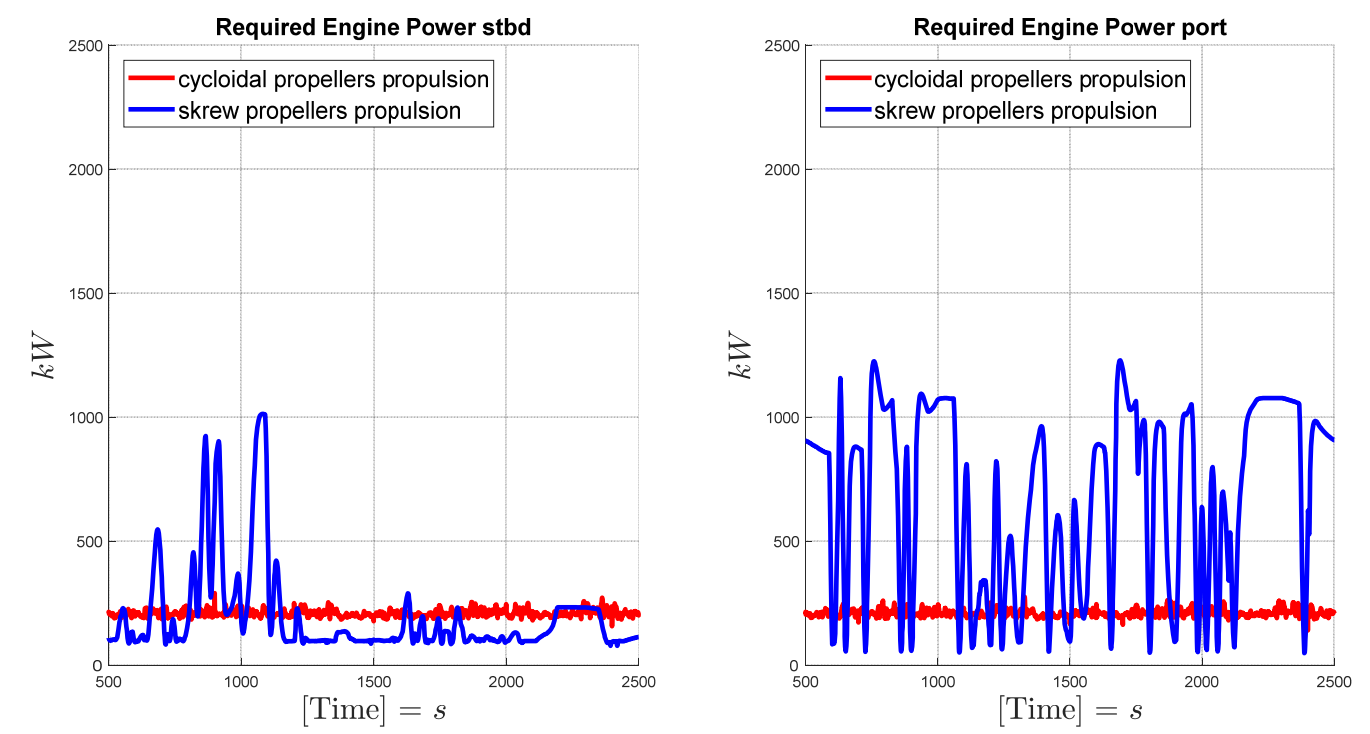

Figure 12. Time history of required engine power for wind speed of $10 \mathrm{kn}$ 

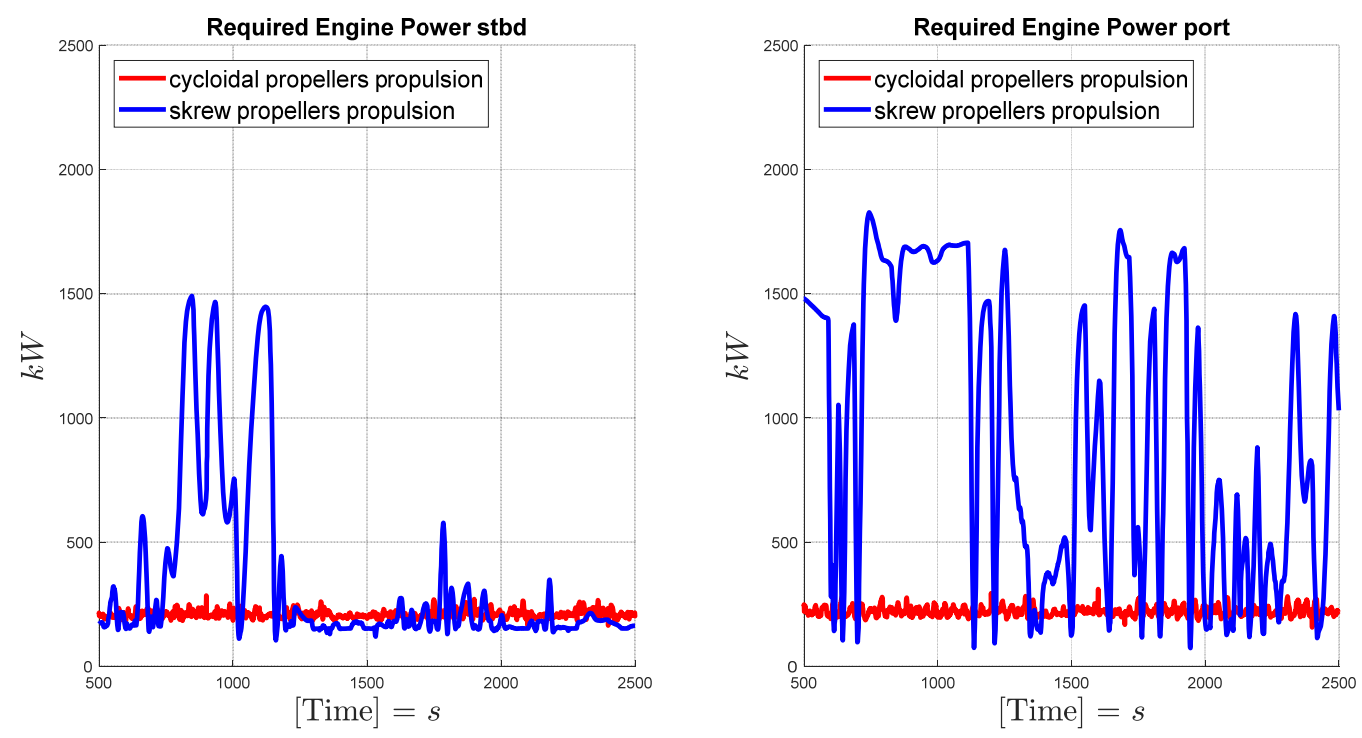

Figure 13. Time history of required engine power for wind speed of $30 \mathrm{kn}$

\section{CONCLUSIONS}

A simulation model for dynamic positioning of a vessel equipped with cycloidal propellers has been presented. Dynamic simulations have been carried out and compared with those regarding the same ship equipped with conventional propellers. The obtained results confirm the conclusions of a previous work (Altosole et al. 2017), where simulations in static conditions were performed. As it was expected, it is shown that cycloidal propellers can be a valid alternative to traditional propellers in case of strong DP requirements. Simulation results have been provided also to illustrate the effectiveness of the proposed propulsion plant and the corresponding thrust allocation, as well as the reliability of the mathematical and numerical model implemented for cycloidal propellers. Future works will concern dynamic simulations of maneuvering at design speed.

\section{REFERENCES}

Alessandri, A., Chiti, R., Donnarumma, S., Luria, G., Martelli, M., Sebastiani, L., Vignolo S. 2014. Dynamic Positioning system of a vessel with conventional propulsion configuration: Modeling and Simulation. Proceedings of MARTECH 2014, 2th International Conference on Maritime Technology and Engineering, Lisbon, Portugal, 1517 October 2014, 725-733.

Altosole, M., Benvenuto, G., Figari, M. 2005. Performance prediction of a planing craft by dynamic numerical simulation. Proceedings of HSMV 2005, 7th Symposium on High Speed Marine Vehicles, Naples, Italy, 21-23 September 2005, pp. 105-111.

Altosole, M., Figari, M, Martelli, M. 2012. Time-domain simulation for marine propulsion applications. Proceedings of SCSC 2012, 2012 Summer Computer Simulation Conference, Genoa, Italy, 8-11 July 2012, 36-43.
Altosole, M., Donnarumma, S., Spagnolo, V., Vignolo, S. 2017. Marine cycloidal propulsion modelling for DP applications. Proceedings of Marine 2017, 7th International Conference on Computational Methods in Marine Engineering, Nantes, France, 15-17 May 2017, 206-219.

Bose, N. 2008. Marine Powering Prediction and Propulsors. The Society of Naval Architects and Marine Engineers.

Donnarumma, S., Martelli M. and Vignolo S. 2015. Numerical models for ship dynamic positioning. Proceedings of Marine 2015, 6th International Conference on Computational Methods in Marine Engineering, Rome, Italy, 1517 June 2015, 1078-1088.

Donnarumma, S., Figari, M., Martelli, M., Vignolo, S., Viviani, M. Design and Validation of Dynamic Positioning for Marine Systems: A Case Study. IEEE Journal of Oceanic Engineering, DOI: 10.1109/JOE.2017.2732298, in press.

Esmailian, E., Ghassemi, H., Heidari, S.A., 2014. Numerical investigation of the performance of voith schneider propulsion, American Journal of Marine Science, Vol. 2, No. 3, 58-62.

Fossen, T.I., 2002. Marine Control System. Marine Cybernetics. Norway: Trondheim.

Fossen, T.I., Sagatun, S.I., Sørensen, A.J. 1996. Identification of dynamically positioned ships, Control Engineering Practice, Vol. 4, Issue 3, 369-376.

Johansen, T.A. \& Fossen, T.I., 2013. Control Allocation-A Survey. Automatica Vol. 49, 1087-1103.

Jürgens, D. \& Moltrecht, T. 2002. Enhanced cycloidal propulsion. The International Workboat Show, New Orleans.

Martelli, M. 2015. Marine propulsion simulation. Berlin: Walter de Gruyter GmbH.

Sørensen, A.J., Sagatun, S.I., Fossen, T.I. 1996. Design of a dynamic positioning system using model-based control, Control Engineering Practice, Vol. 4, Issue 3, 359-368.

Sørensen, A.J. 2011. A survey of dynamic positioning control systems, Annual Reviews in Control, Vol. 35, Issue 1, 123-136.

Taniguchi, K. 1962. Sea Analysis of the vertical axis propeller. Proc. of the 4th symposium on Naval Hydrodynamics, Office of Naval Research, Washington. 